\title{
LA RESPONSABILIDAD SOCIAL UNIVERSITARIA Y EL DESARROLLO LOCAL: EL ROL DE LA UNIVERSIDAD NACIONAL DEL NORDESTE
}

\author{
GABRIEL GELMAN \\ MENCIÓN ESPECIAL - DOCENTE - \\ CONCURSO $60^{\circ}$ ANIVERSARIO
} FACULTAD DE CIENCIAS ECONÓMICAS - UNNE 


\section{RESUMEN}

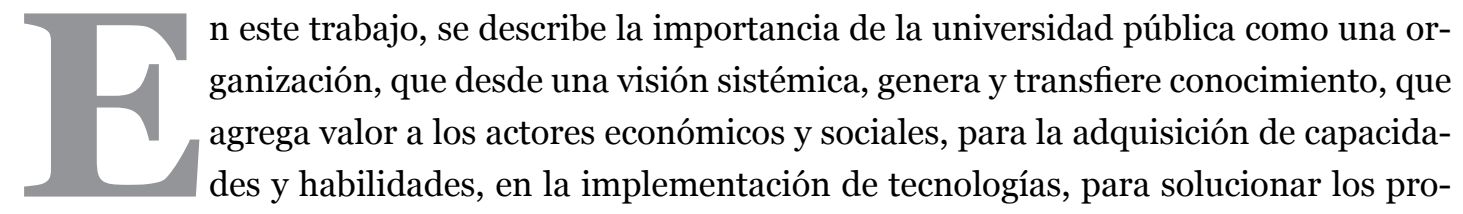
blemas multidimensionales del desarrollo local.

Además, se propone a la planificación estratégica como una herramienta administrativa, que permite articular las acciones de responsabilidad social con el desarrollo local. Se describe la dinámica de la UNNE, en cuanto a su responsabilidad social y su vinculación con el medio.

\section{INTRODUCCIÓN}

El problema primordial que enfrenta nuestra región es la pobreza, efecto de numerosas crisis y de la mala administración implementada en nuestro país.

Sin dudas para enfrentar el problema de la pobreza y lograr desarrollo económico y social, se deben implementar políticas y estrategias que permitan mejorar la competitividad de la región.

Si consideramos que en el actual paradigma, para ser competitivos, no basta con la reducción de costos, y las estrategias deben ser ofensivas, cobrando fundamental importancia las ventajas competitivas dinámicas, basadas en la innovación de proceso, innovación de producto, e innovar en la organización de las empresas (Neffa, 2002). ${ }^{1}$

Es en este paradigma, donde la universidad pública, dentro de sus políticas de responsabilidad social, ejerce un rol fundamental de liderazgo en los procesos de mejora de la competitividad y desarrollo local, a través de la transferencia de tecnologías, que permiten generar fuerzas sinérgicas para el desarrollo local, en su aspecto económico y social.

La UNNE considera, en el marco de su visión estratégica, su responsabilidad social, desarrollando acciones que agreguen valor y mejoren el capital social de su entorno a través de la transferencia de conocimientos a las organizaciones del medio, que perfeccionen sus procesos y sus productos, provocando mejoras en la competitividad de la región y desarrollo económico y social sustentable en el tiempo.

\section{VISIÓN SISTÉMICA DE LA UNIVERSIDAD}

Partimos de que "Un sistema es un conjunto de elementos interrelacionados. Por tanto, un sistema es una entidad que se compone al menos de dos elementos y una relación que es válida entre cada uno de sus elementos y al menos otro elemento del conjunto. Cada uno de 
los elementos de sistema está relacionado de los elementos restantes de manera directa o indirecta. Además, ningún subconjunto de elementos deja de estar relacionado con cualquier otro subconjunto" (ACKOFF; 1974). ${ }^{2}$

Consideramos a la Universidad como un sistema que produce, recibe recursos del medio y utiliza diferentes tecnologías de transformación que responden a los objetivos y estrategias de la organización, para brindar sus productos al medio en el cual está inserta.

El sistema de la Universidad tiene limites permeables a través de los cuales se intercambia permanentemente información que sirve como retroalimentación para que los productos que genere la organización, sean pertinentes a las necesidades del medio en el cual la misma está inserta.

La gestión del conocimiento, orientado a mejorar el capital social del entorno, es uno de los subsistemas de la universidad, que debe pivotear en un modelo estratégico, situacional, con una mirada permanente en su contexto.

Se debe destacar la importancia de la visión sistémica en esa relación de la Universidad con los actores del contexto, por cuanto, permite articular la asociatividad entre actores públicos y privados y sobre todo la sinergia que se genera como una característica importante de los sistemas sociales abiertos.

\section{DESARROLLO LOCAL}

El desarrollo es abarcativo de varias dimensiones: económica, política, social, ambiental, cultural, las que deben ser contempladas dentro de un modelo sistémico que nos permita entender el comportamiento de las partes, sin perder de vista el todo, es decir, integrar el análisis y la síntesis.

Si hablamos de desarrollo, debemos asumir la idea de algo complejo, difícil de explicar, si se adopta una visión lineal de la realidad.

Giovanni Reyes nos plantea que "Un sentido con mayor aplicabilidad y concreción en la definición de desarrollo establecería que el mismo está caracterizado por condiciones en las cuales los bienes y servicios se encuentran crecientemente al alcance de los grupos sociales que conforman la sociedad. Esta característica implicaría una mayor integración social y económica dentro de las sociedades, y por ello se disminuiría la existencia de grupos viviendo en condiciones de marginalidad. Por otra parte el desarrollo establecería una condición de acceso a los servicios sociales y a la participación social activa. En el primero de los casos se hace referencia a los sistemas de educación, y a la satisfacción de las necesidades de sobrevivencia en términos de alimento, vivienda, vestido, salud y seguridad. En el caso de la participación social activa se refiere a la capacidad del individuo y de las instituciones sociales a garantizar que las agrupaciones de poder sean instancias de intermediación entre los sujetos y actores en la toma de decisiones”. ${ }^{3}$

\footnotetext{
${ }^{2}$ Ackoff, Rusell (1974). El paradigma de Ackoff: una administración sistémica. Buenos Aires: Editorial Limusa.

${ }^{3}$ Reyes, Giovanni E. Comercio y Desarrollo: Bases Conceptuales y Enfoque para América Latina y el Caribe, www.zonaeconomica. com/desarrollo-comercio
} 
La importancia de la visión sistémica del desarrollo local, es tomada por el prestigioso autor Manuel Alburquerque ${ }^{4}$ que menciona en su Manual del Agente del Desarrollo Local, “...que la recurrente simplificación macroeconómica, y el habitual enfoque sectorial de la economía nacional, no promueven una visión novedosa, en la cual la economía nacional se contempla no sólo como un conjunto de sectores, sino también como un conjunto de sistemas económicos locales. Si esto sucede en los países desarrollados, en el caso latinoamericano la situación es aún más incipiente, porque ni siquiera existe un esfuerzo de investigación sistémico acerca de las propias experiencias de desarrollo económico local en curso. Esta tarea, aún pendiente, podría ayudar a deducir orientaciones solventes en este campo...”.

Vázquez-Barquero, define el desarrollo local: "Un proceso de crecimiento económico y de cambio estructural que conduce a una mejora en el nivel de vida de la población local, en el que se pueden identificar tres dimensiones: una económica, en la que los empresarios locales usan su capacidad para organizar los factores productivos locales con niveles de productividad suficientes para ser competitivos en los mercados; otra, sociocultural, en que los valores y las instituciones sirven de base al proceso de desarrollo; y, finalmente, una dimensión políticoadministrativa en que las políticas territoriales permiten crear un entorno económico local favorable, protegerlo de interferencias externas e impulsar el desarrollo local". ${ }^{5}$

\section{LA ADMINISTRACIÓN DEL DESARROLLO LOCAL}

"La administración tiene vinculación con el desarrollo, la dependencia, las relaciones económicas internacionales, el nivel de educación, y el nivel tecnológico”. ${ }^{6}$

Se entiende por desarrollo no sólo el crecimiento cuantitativo de variables económicas sino el resultado de la implementación del proceso administrativo, como herramienta estratégica para el logro de los objetivos sociales de una comunidad, para generar un mejor nivel de vida en la misma.

Administrar el desarrollo local es, el conjunto de conocimientos que respaldan la gestión para el desarrollo local. Como proceso, es: planificar, organizar, dirigir y controlar, actividades y recursos para el logro de los objetivos y metas fijados en los procesos de planificación para el desarrollo local.

Se genera en los niveles altos de decisión gubernamental, una cadena de fines y medios descendente, que debe complementarse con una de dirección ascendente, “desde abajo", donde se prioricen las miradas de los actores locales, en un proceso sinérgico, donde el Municipio cumple un papel fundamental como articulador y promotor de acciones de cooperación e

\footnotetext{
${ }^{4}$ Alburquerque, F. (1995). Manual del Agente de desarrollo local. Santiago de Chile: Ediciones Sur.

${ }^{5}$ Vazquez-Barquero A. (1988), Desarrollo local. Una estrategia de creación de empleo. Madrid: Editorial Pirámide.

${ }^{6}$ Álvarez,H(2003).Administración. Cordoba: Eudeba.1988
} 
integración entre el ámbito público y privado, que contemplen el desarrollo de industrias, la integración y cooperación estratégica entre empresas, el fomento de la actividad emprendedora, el fortalecimiento de las micro y pequeñas empresas, el desarrollo de servicios sociales y culturales a partir de sus propios recursos humanos y materiales, etc. Este esfuerzo a partir de lo propio, es lo que se denomina desarrollo endógeno.

Para Garofoli “Desarrollo endógeno significa, en efecto, la capacidad para transformar el sistema socio - económico; la habilidad para reaccionar a los desafíos externos; la promoción de aprendizaje social; y la habilidad para introducir formas específicas de regulación social a nivel local que favorecen el desarrollo de las características anteriores. Desarrollo endógeno es, en otras palabras, la habilidad para innovar a nivel local". ${ }^{7}$

Se gestiona el desarrollo local en todos los niveles de la realidad local, integrando a las diferentes organizaciones, con una visión sistémica, integrante, proactiva ante los cambios, con herramientas, que permitan enfrentar lo complejo del desarrollo local.

\section{PLANIFICACIÓN DEL DESARROLLO LOCAL}

Planificar, como una de las funciones del proceso administrativo, es prever acciones que permitan posicionar a la organización ventajosamente en el sector en el cual desarrolla su actividad. Se fijan objetivos y metas que guían la acción y se formulan operaciones que permitan el logro de dichos objetivos. Es un proceso que se basa en la toma de decisiones. Se eligen objetivos, cursos de acción, productos, segmentos de mercado, estrategias, etc.

Las operaciones utilizan recursos para poder ser llevadas a cabo, y deben ser realizadas en tiempos determinados y se deben establecer responsables a cargo de las mismas.

"La planificación y gestión estratégica demuestran ser herramientas idóneas y eficaces para establecer y construir una estrategia de desarrollo local. Esto es, un proceso de mejora continua y sistemática de la productividad y competitividad del sistema productivo-territorial local, mediante el aprovechamiento pleno de los potenciales endógenos y los recursos e impulsos exógenos. Todo ello de cara a lograr la eficiencia, la equidad y la preservación del entorno medio ambiental".

El Planeamiento Estratégico define las grandes líneas de acción, la estrategia de desarrollo local, es decir los caminos que se deben transitar para el logro de los principales propósitos de la región, alineados con el plan global y los planes sectoriales, movilizando todos o la mayor parte de los recursos locales disponibles.

Para llevar a cabo una eficaz implementación de la administración del desarrollo local, se requiere desarrollar primero un proceso de planificación estratégica, que permita a la gerencia

\footnotetext{
${ }^{7}$ GARAFOLI, G. (1995), "Desarrollo económico, organización de la producción y territorio", en A. Vázquez-Barquero y G. Garafoli (edits.) Desarrollo Económico Local en Europa, Colegio de Economistas de Madrid, Colección. Economistas Libros.

${ }^{8}$ Cotorruelo Menta, R. (1996), Planificación y gestión estratégica del desarrollo local. Madrid: Cuadernos Aguilar, INEM, FUAM.
} 
pública, a partir de un diagnóstico situacional, donde se analicen las oportunidades y amenazas para cotejarlas con las capacidades fundamentales locales para establecer, luego, los objetivos estratégicos, relativos al contexto histórico, y los resultados que serán evaluados y que expresen la medida del logro de los mismos, los objetivos correspondientes, quedando conformada así la jerarquía y red de fines y metas, que como una verdadera carta de navegación, guiarán las operaciones para el desarrollo local y son el principal producto del proceso de planificación estratégica y a la vez insumo fundamental del sistema de administración pública por resultados.

\section{LA PLANIFICACIÓN ESTRATÉGICA COMO UNA HERRAMIENTA DE ARTICULACIÓN ENTRE LA RESPONSABILIDAD SOCIAL UNIVERSITARIA Y EL DESARROLLO LOCAL}

Los objetivos formulados en el proceso de planificación estratégica del desarrollo local, priorizados y jerarquizados, son insumos para las acciones de responsabilidad social de las universidades, que buscan elevar el capital social local. Esto se logra, principalmente, priorizando una eficaz y eficiente gestión del conocimiento que transforme las experiencias e información en lecciones aprehendidas, en un proceso de retroalimentación sistemático que permita monitorear los efectos de las acciones llevadas a cabo por la universidad y que impactan en su entorno. (Ver figura 1)

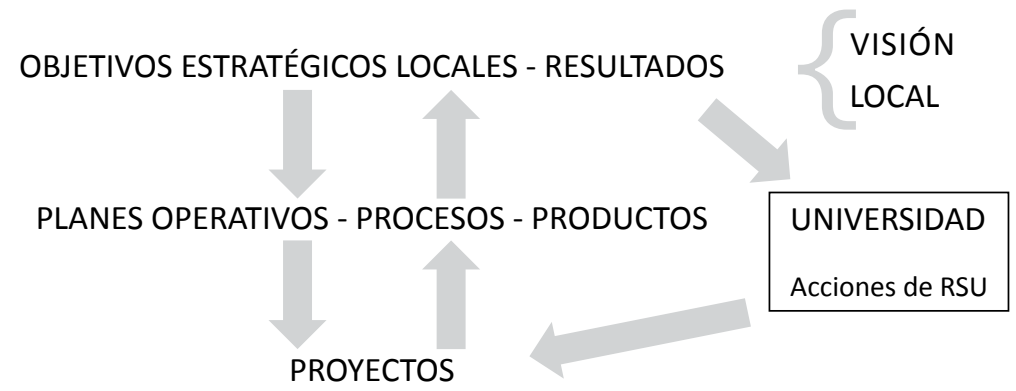

Figura 1. Elaboración propia.

La universidad se presenta como una organización generadora de conocimiento que construye capital social, adquiriendo los actores económicos y sociales, capacidades y habilidades en la implementación de tecnologías para solucionar los problemas multidimensionales del desarrollo local.

Para Kliksberg (2005), uno de los mayores desafíos de la Universidad actual, transita no por influir solamente en los acontecimientos sociales, sino en construir procesos sociales con identidad regional sostenibles que aseguren un verdadero empoderamiento desde la base. ${ }^{9}$

Se puede observar cómo, en El trabajo sobre ética y capital social del BID/SDS (2006), se pretende impulsar la definición de políticas de responsabilidad social que hagan de la Uni- 
versidad un centro para la formación de profesionales socialmente responsables con las comunidades que los rodean y fomenten el tejido social que requiere la sociedad para promover la apertura a nuevos proyectos educativos y nuevas formas de gestión que aseguren una adecuada respuesta a las necesidades de la sociedad logrando su desarrollo social y sostenible. ${ }^{10}$

\section{LA RESPONSABILIDAD SOCIAL DE LA UNNE Y EL DESARROLLO LOCAL}

Las acciones de responsabilidad social de la UNNE están encuadradas en su sistema de planificación estratégica, porque se alinean con la visión estratégica de la organización, que busca que esas acciones tengan efectos beneficiosos en su entorno y que sean sostenibles en el tiempo. (Ver figura 2)

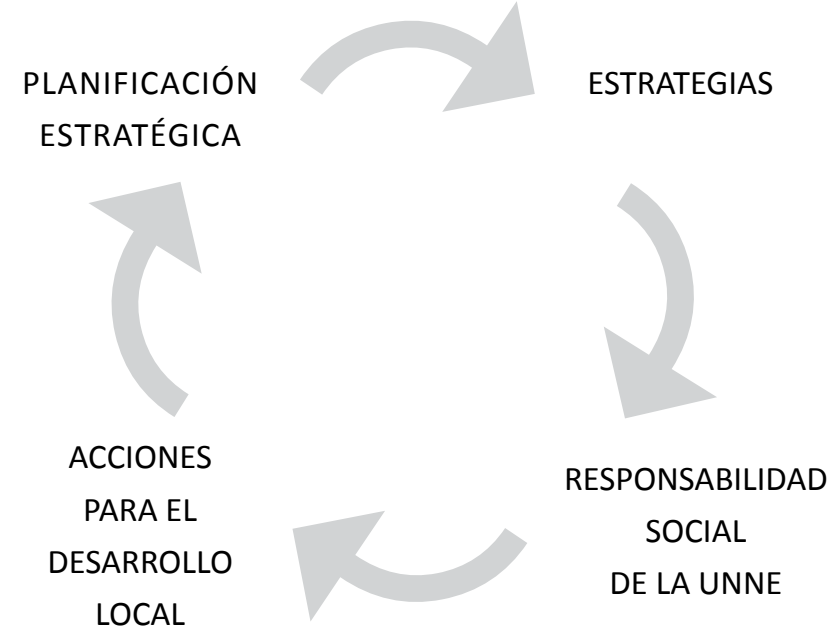

Figura 2. Elaboración propia.

Algunos ejemplos que demuestran cómo la Universidad Nacional del Nordeste, en sus políticas, estrategias, programas y acciones, explicita un comportamiento socialmente responsable:

a) Sistema de Evaluación Permanente ${ }^{11}$

"La evaluación de la universidad se sustenta en una concepción holística de la institución a partir de la cual la autoevaluación es requisito y recurso insoslayable para respetar la diversidad, aumentar el compromiso de sus miembros y favorecer la integración de las contribuciones, a los efectos de potenciar el logro de los fines y la vinculación con el entorno.

\footnotetext{
${ }_{10}$ III Diálogo Global sobre Responsabilidad Social Universitaria, a través de la Iniciativa Interamericana de Capital Social, Ética y Desarrollo del BID/SDS. Febrero 2006.

${ }^{11}$ Extraído de la WEB de la UNNE www.unne.edu.ar
} 
El desarrollo de los procesos evaluativos está orientado por los principios básicos de la Universidad pública uno, uno de los cuales es:

- Responsabilidad social: responder por la calidad y la cantidad de sus actividades y productos educativos en función de las necesidades y de los valores públicos”.

b) El Programa Nacional de Formación en Ética para el Desarrollo por un acuerdo con la Universidad de Buenos Aires se dicta en la UNNE en el marco del 60 aniversario de la Facultad de Ciencias Económicas.

"Dicho programa apunta a formar una nueva generación de jóvenes a nivel nacional y regional conscientes de la necesidad de desarrollo local con ética y compromiso social. Para ello, a través de un ciclo de charlas sobre temas diversos, el Programa de Formación intenta difundir la premisa de que el desarrollo es más amplio que el crecimiento económico, debido a que el objetivo final debe ser el bienestar social".

En dicho marco, El Secretario General Académico de la UNNE, profesor Cristian Piris, destacó la importancia de la responsabilidad social empresaria como política institucional. El profesor disertó ante un nutrido grupo de estudiantes avanzados, docentes y profesionales en el Salón de Uso Múltiples de la Facultad de Ciencias Económicas. "La responsabilidad social universitaria es un término amplio, aún en consolidación, pero que la UNNE ha decidido adoptarla como guía de su gestión como lo han hecho las principales instituciones de Educación Superior" resaltó el funcionario académico. ${ }^{12}$

c) La UNNE, articula permanentemente acciones con actores sociales relevantes locales, que tienen como eje la responsabilidad social. En este marco, “...el Rector de la Universidad Nacional del Nordeste, ingeniero Eduardo E. del Valle recibió la visita del Arzobispo de la Diócesis de Corrientes, Monseñor Andrés Stanovnik". En la reunión Ambos referentes departieron cordialmente durante aproximadamente una hora sobre temas relacionados con la actualidad social, religión y universidad, sin dejar de lado las acciones de responsabilidad social que les cabe ejecutar y promover desde el lugar de liderazgo que ocupan.

En este aspecto, el Rector del Valle interiorizó al Arzobispo sobre la totalidad de programas que en este 2011 desarrollará la Universidad Nacional del Nordeste en distintas comunidades de Chaco y Corrientes, en el marco del reconocido programa social UNNE Salud impulsado por esta Casa de Altos Estudios. Al respecto, el titular de la UNNE hizo especial hincapié en la nueva concepción institucional adoptada por la Universidad en esta nueva etapa que le toca presidir, que estará estrechamente vinculada con la comunidad a través de acciones de extensión concebidas para cubrir las necesidades de la sociedad de influencia.

Monseñor Stanovnik enfatizó en el encuentro su reconocimiento a la Universidad Nacional del Nordeste como institución de educación superior señera en la región y la trascendente importancia de extender los vínculos para delinear acciones futuras en beneficio de la 
comunidad"13.

d) La UNNE, a través de sus múltiples investigaciones, colabora en la sistematización del estudio de las potencialidades económicas de nuestra región lo cual genera que se pueda llegar a vincular las necesidades sociales y económicas con las capacidades productivas y con la educación.

Una de las investigaciones en marcha es "ADMINISTRACIÓN DE AGLOMERACIONES COMPETITIVAS Y CADENAS DE VALOR PARA EL DESARROLLO LOCAL EN LAS PROVINCIAS DE CHACO Y CORRIENTES" ${ }^{14}$

Los objetivos de dicha investigación son:

Aportar un diagnóstico estratégico preciso sobre la aplicación de la administración para el desarrollo local en las principales aglomeraciones competitivas, con las siguientes especificaciones:

a) Identificar aglomeraciones (de empresas y otras instituciones) con significativa generación de valor, fundamentalmente, económico.

b) Precisar sus actores, perfiles y vinculaciones.

c) Precisar las fuerzas competitivas que las caracterizan.

d) Determinar sus aportes al desarrollo humano y calidad de vida, sobre bases locales.

e) Identificar la aplicación del proceso administrativo para la gestión competitiva de cada aglomeración. Esto es, si hay planificación, organización, dirección, control y una retro alimentación ordenada al desarrollo humano y competitivo de cada aglomeración.

f) Caracterizar la aplicación (o no) de cada una de las funciones esenciales de la administración en cada aglomeración.

g) Caracterizar la aplicación (o no) del ciclo estratégico para la administración en cada aglomeración.

h) Precisar el diagnóstico de cada aglomeración en orden al desarrollo humano y sustentable, sobre bases locales.

i) Modelizar el comportamiento de cada aglomeración de impacto significativo sobre el desarrollo humano local.

La posibilidad de lograr la cooperación estratégica de empresas, a través de cadenas productivas y el fortalecimiento de las micro, pequeñas y medianas empresas, son los ejes estratégicos que se siguen para poder lograr el crecimiento y desarrollo local.

\section{CONCLUSIONES}

Definir de forma participativa y consensuada cómo se debe vincular lo social con lo productivo y lo educativo, es trascendente para todas las localidades de nuestra región.

En este nuevo contexto de transformaciones sociales y productivas de la región nordeste de nuestro país, surge la posibilidad de incorporar nuevos actores a este proceso, entre ellos

\footnotetext{
${ }^{13}$ Extraído de : www.unne.edu.ar

${ }^{14}$ Información suministrada por la cátedra Principios de administración. Facultad de Ciencias Economicas.UNNE.
} 
la Universidad Pública y dentro de ésta a sus recursos humanos, nucleados en sus diferentes áreas del conocimiento, en todas sus ramas.

Desde la Universidad Nacional del Nordeste, se intenta cumplir un rol como agente de desarrollo local, adquiriendo progresivamente mayores capacidades para participar en el diseño e implementación de experiencias de desarrollo, ejerciendo una actitud proactiva sobre su entorno. Esta institución promueve la articulación de los recursos de las diferentes localidades de la región, genera y transfiere conocimiento, y promueve el fortaleciendo de modelos asociativos de los actores económicos y sociales locales.

La UNNE tiene un rol muy activo en el diseño de estrategias que motorizan y dan marco a la implementación de planes de acción gestionados desde lo local.

La UNNE es un actor que participa activamente en los procesos de planificación estratégica del desarrollo local, colaborando en la organización de las estructuras que son el marco para llevar a cabo las acciones de desarrollo planeadas, coordinando e integrando los distintos niveles institucionales que participan del proceso de toma de decisiones políticas económicas y sociales y monitoreando la implementación de los planes y programas, que permiten el logro de la políticas públicas para el desarrollo local.

La propuesta desde la UNNE, en el marco de su responsabilidad social, a través de la generación y transferencia de conocimiento, para aumentar el capital social local, es asumir un papel protagónico, generando los cambios necesarios, para posibilitar mejorar la realidad económica y social de nuestra región a través de:

- Estimular la planificación concertada con actores locales y / o regionales.

- Articular el sistema de educación superior y la capacitación con el perfil productivo local.

- Incentivar la eficiencia y la eficacia en la gestión pública y privada local.

- Acrecentar el valor los recursos endógenos existentes en nuestra región.

- Sistematizar la información entre los gobiernos locales, con el objetivo de poder implementar políticas públicas acordes con la realidad productiva local.

\section{REFERENCIAS BIBLIOGRAFICAS}

Ackoff, Rusell (1974). El paradigma de Ackoff: una administración sistémica. Buenos Aires: Editorial Limusa.

Alburquerque, F. (1995). Manual del Agente de desarrollo local. Santiago de Chile: Ediciones Sur.

Álvarez, H. (2003). Administración. Cordoba: Eudeba. 
Cotorruelo Menta, Romeo (1996), Planificación y gestión estratégica del desarrollo local. Madrid: Cuadernos Aguilar, INEM, FUAM.

Garafoli, G. (1995), "Desarrollo económico, organización de la producción y territorio", en A. Vázquez-Barquero y G. Garafoli (edits.) Desarrollo Económico Local en Europa. Madrid: Colegio de Economistas de Madrid, Colección. Economistas Libros.

Kliksberg, B. (2005), “La Responsabilidad Social Universitaria” Buenos Aires: Programa PNUD.

Vazquez-Barquero A. (1988), Desarrollo local. Una estrategia de creación de empleo. Madrid: Editorial Pirámide.

III Diálogo Global sobre Responsabilidad Social Universitaria, a través de la Iniciativa Interamericana de Capital Social, Ética y Desarrollo del BID/SDS. Febrero 2006.

\section{INTERNET}

Neffa, J. C. (2002): "Situemos el problema de la competitividad en su contexto histórico internacional”, ftp://ftp.secyt.gov.ar/pub/Web/competitividad_Panel1_Neffa.pdf

Reyes, Giovanni E. Comercio y Desarrollo: Bases Conceptuales y Enfoque para América Latina y el Caribe, www.zonaeconomica.com/desarrollo-comercio

www.diarionorte.com

www.unne.edu.ar

\section{CURRÍCULUM VITAE}

GABRIEL GELMAN

Licenciado en Administración, egresado de la Universidad Nacional del Nordeste.

Profesor de las Cátedras de Administración Pública, del Curso de Nivelación Módulo de Administración y de Principios de Administración en TAGIU en la Facultad Ciencias Económicas de la UNNE; en Ciencia Política y de Gobierno de la Universidad en Ciencias Sociales y Empresariales; de Organización Administrativa de la Fundación de la UNNE.

Secretario del Departamento de Administración de la Facultad de Ciencias Económicas de la UNNE.

licenciadogabrielgelman@yahoo.com.ar 